\title{
Fundamentals of Debugging Using a Resolution Calculus ${ }^{\star}$
}

\author{
Daniel Köb and Franz Wotawa \\ Technische Universität Graz, \\ Institute for Software Technology, \\ 8010 Graz, Inffeldgasse 16b/2, Austria \\ $\{$ koeb, wotawa\}@ist.tugraz.at \\ http://www.ist.tugraz.at/
}

\begin{abstract}
Detecting and localizing a fault within a program is a nontrivial and time consuming task. Most of the efforts spent for automating the task have focused on fault detection. In this paper we shift the focus on fault localization. We introduce a resolution calculus that allows for representing the program's behavior based on correctness assumptions. The fault localization task is reduced to finding consistent assumptions which are represented as a non-monotonic reasoning process where efficient algorithms exist. Finally, we compare our approach with a previous approach to fault localization that is based on trace analysis. As a result we can show that our approach is less sensitive to search assumptions.
\end{abstract}

\section{Introduction}

Detecting, locating, and correcting bugs in programs are time consuming and expensive tasks and are summarized under the term debugging. Currently, debugging is hardly automated with some rare exceptions. For example in fault detection, tools for automated verification and test case generation have been available. However, there have been almost no tools and techniques for automated fault localization presented so far. In this paper we introduce foundations for automated debugging which are based on the program's semantics. The underlying idea is to directly use the program's behavior for a given test case to derive the location of a bug. For this purpose we represent the behavior of a program in terms of a calculus and the test cases as predicates at certain lines in the code. Moreover, we make underlying assumptions about the state of statements explicit. This state can be either bug-free (not abnormal) or faulty (abnormal). These explicit assumptions are used to modify the program's behavior and to check the behavior against the given test case. For example, if we assume a statement to be faulty and all others to be correct, then we might not be able anymore to derive a value for a variable at a certain position in the code which contradicts a given test case. Hence, we know that the assumption is consistent

\footnotetext{
* The work presented in this paper has been supported by the Austrian Science Fund (FWF) grants P15265-INF and P17963-INF. Authors are listed in alphabetical order.
} 
with the given test case and as a consequence that assuming the statement to be faulty explains differences between the intended behavior given by the test case and the detected behavior given by the program run. Because the semantics of a program, i.e., a model of the program's behavior, is directly used to identify the fault location, our approach is called model-based debugging. We illustrate the basic idea behind model-based debugging using the following tiny program, which implements the computation of the circumference $c$ and the area a of circle from a given radius $r$.
1. $\mathrm{d}=2 * \mathrm{r}$;
2. $c=d * 3.14$;
3. $\mathrm{a}=\mathrm{d} * \mathrm{~d} * 3.14$;

Obviously, the program contains a bug in line 3 . We further assume $r$ to be 1 before line 1 , and c to be 6.28 and a to be 3.14 after line 3 . This test case can be represented in terms of predicates for certain positions in the code. For our example, we have the following situation:

$$
\begin{array}{ll} 
& \{r=1\} \\
1 . & \mathrm{d}=2 * \mathrm{r} ; \\
2 . & \mathrm{c}=\mathrm{d} * 3.14 ; \\
3 . & \mathrm{a}=\mathrm{d} * \mathrm{~d} * 3.14 ; \\
& \{c=6.28 \wedge a=3.14\}
\end{array}
$$

We apply Hoare's calculus [8] to propagate predicates through the program. Propagating predicates forwards is always possible by applying a statement's semantics, whereas backwards propagation of predicates is not possible for all statements. Especially heap manipulating statements that produce garbage prevent backwards propagation. In particular we use only the rules for program statements and assignments:

$$
\{P(e / x)\} x=e\{P\} \quad \frac{\{P\} s_{1}\{Q\} ;\{Q\} s_{2}\{R\}}{\{P\} s_{1} ; s_{2}\{R\}}
$$

It is worth noting that these rules can only be used when assuming statements to be bug-free. Because if a statement is assumed to be faulty, we do not know anything about its correct behavior. But even with limited backwards propagation of predicates we are able to derive worthwhile information for debugging. Using these rules and assuming all statements to be correct we obtain the following logical sentences:

$$
\begin{array}{ll} 
& \{r=1 \wedge 6.28=2 \cdot r \cdot 3.14 \wedge 3.14=2 \cdot r \cdot 2 \cdot r \cdot 3.14\} \\
1 . & \mathrm{d}=2 * \mathrm{r} ; \\
& \{6.28=d \cdot 3.14 \wedge 3.14=d \cdot d \cdot 3.14\} \\
2 . & \mathrm{c}=\mathrm{d} * 3.14 \\
& \{c=6.28 \wedge 3.14=d \cdot d \cdot 3.14\} \\
3 . & \mathrm{a}=\mathrm{d} * \mathrm{~d} * 3.14 \\
& \{c=6.28 \wedge a=3.14\}
\end{array}
$$

Obviously the sentences before line 1 and between lines 1 and 2 are unsatisfiable because it is not possible to derive different values for the same variables 
at a specific position in the code. Because of the detected inconsistency the underlying assumption of all statements being bug-free cannot be correct. Hence, we have to change the assumptions. If we assume statement 1 to be faulty, we still derive an inconsistency. Moreover, when assuming a fault in statement 2, we again come to an unsatisfiable logical sentence. The argumentation is as follows:

From $r=1$ and statement 1 we derive $d=2$. From the given predicates at the end of the program we derive predicates before line 3 .

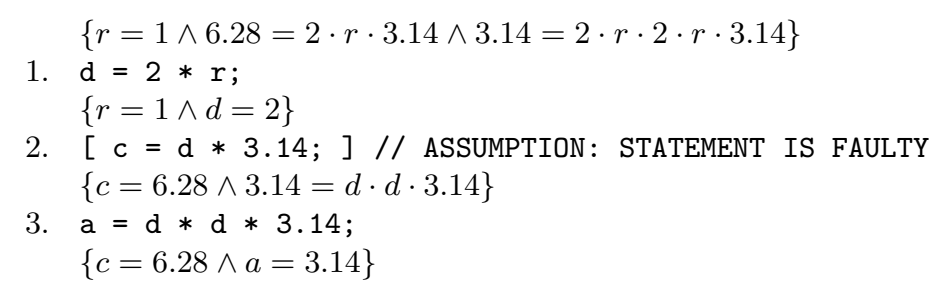

However, we do not know anything about the behavior of the statement in line 2. To allow for deriving new predicates before and after the statement, we introduce a new assumption saying that Hoare's rules can be applied to predicates that do not contain the target variable of a statement that is assumed to be faulty. If using this assumptions, we finally again obtain an inconsistency and we know that statement 2 is no explanation for the misbehavior.

In summary we conclude that the correctness assumptions for statements 1,3 and 2,3 both lead to an inconsistency. Such assumptions that lead to inconsistencies are called conflicts. In order to remove both conflicts, we have either to assume statements 1,2 or statement 3 to be incorrect and the rest of the statements to be correct. Both of the statement sets are explanations to the misbehavior but the assumption that statement 3 contains a bug is the smallest explanation. Hence, using the semantics of the statements and a test case allows to compute a diagnosis, i.e., statements that when assumed to contain a bug explain differences between the program's behavior and the given test case.

The objectives of the paper are: (1) to introduce a formal model for program debugging that is based on well known paradigms, (2) to present the formal properties that follow from the given definitions, (3) to compare the outcome of the proposed methodology with other debugging techniques, and (4) to discuss limitations and related research.

The paper is organized as follows. We first introduce the basic definitions of model-based debugging. In the following section we briefly introduce the basic definitions of another approach to fault localization which is based on program traces. Afterwards we present a case study which compares the outcome of both approaches. Finally, we discuss related research and conclude the paper.

\section{Model-Based Debugging}

In the introduction we used an example to illustrate the basic idea behind modelbased debugging (MBD). It is important for MBD to have a logical program model and a test case. The model in our case is a calculus that is based on 
Hoare's calculus with some slight modifications. The modifications are due to the assumptions about the state of a statement to be either faulty or bug-free. These explicit assumptions are used to compute diagnoses. In the first part of this section we discuss the logical model for debugging. The second part is devoted to the definition of debugging and some of its consequences. The underlying definitions except the modeling of the program's behavior originate from modelbased diagnosis 9] but are adapted to closely represent the fault localization within programs instead of physical systems.

The first step in providing the formal foundations of debugging is to state the debugging problem which has to be solved. As explained within the elaboration of our introductory example, we have to know about the program's behavior and about a test case containing the expected variable values at certain positions in the code.

Definition 1 (Debugging problem). Given a program $\Pi$. A debugging problem is a tuple $(\mathcal{D}, \mathcal{T})$ where $\mathcal{D}$ is a set of logical rules, i.e., a calculus, that represents the behavior of the individual statements of $\Pi$, and $\mathcal{T}$ is a set of predicates which state the values of variables at certain positions in program $\Pi$.

The model $\mathcal{D}$ of a given program $\Pi$ in the definition of a debugging problem has to fulfill some requirements. First, the model has to provide means for reasoning about predicates which state the values of variables. Second, it is crucial that the model makes assumptions about the assumed state of a statement explicit. Whenever a statement is assumed to behave correct or incorrect the respective behavior has to be available within the model. Finally, reasoning should not only be from the beginning of a program to its end but also in the opposite direction.

Figure 1 shows a model for a simple imperative language. The $A b \backslash 1$ predicate is used to represent the explicit assumption about the state of a statement, i.e., to be either faulty or bug-free. The latter is represented by the negation of the $A b$ predicate. For simplicity, the model further assumes that every statement of program $\Pi$ has a unique corresponding index and that reasoning can be done in both directions if not otherwise stated.

$$
\begin{gathered}
\neg A b(i) \Rightarrow\{P(e / x)\} x=e\{P\} \\
\frac{\{P\} s_{1}\{Q\} ;\{Q\} s_{2}\{R\}}{\{P\} s_{1} ; s_{2}\{R\}} \\
\neg A b(i) \Rightarrow \frac{\{P \wedge p\} s_{1}\{Q\} ;\{P \wedge \neg p\} s_{2}\{Q\}}{\{P\} \text { if } p \text { then } s_{1} \text { else } s_{2} \text { end if }\{Q\}}
\end{gathered}
$$

$$
A b(i) \Rightarrow\{P\} s\{P\} \text { if } P \text { does not contain any defined variable of statement } s
$$

Fig. 1. Meta-model of debugging calculus D. $i$ denotes the line number of the considered statement. 
Rules (1), (2), and (3) of model $\mathcal{D}$ from Figure 1 originate from Hoare's calculus. Rule (4) is introduced because all information about variable values that are not changed by a certain statement should be propagated to the other statements. Of course there is the underlying assumption behind that the fault does not correspond to any defined variable. For example, if we assume the assignment statement $\mathrm{x}=\operatorname{not}(\mathrm{y})$; to be faulty, then all information about the value of a variable $z$ is passed by. Only information about the value of variable $\mathrm{x}$ is blocked. Note that Rule (4) also captures the case where $s$ is a conditional statement. In this case all variables that are defined in any sub-block are not allowed to occur in predicate $P$.

In order to complete the modeling part, we have to provide a rule for loop statements. The Hoare calculus requires a loop invariant. Computing such an invariant is not always possible and for our purpose not necessary. Since we only consider one specific test case we know how often a loop statement has been executed simply by executing the program. Hence, we replace a loop statement by nested conditional statements and use the model for conditional statements instead. The statement while $p$ do $s_{1}$ end while is represented by if $p$ then $s_{1}$ if $p$ then ... else $\epsilon$ end if else $\epsilon$ end if where $\epsilon$ denotes the empty statement block. A necessary condition for this transformation is that the program halts on the given input. Hence, faults related to infinite loops cannot be handled within our framework.

The second part of the framework comprises the formal definition of debugging and some consequences. Debugging herein is restricted to fault localization. As mentioned in the introductory example we define the result of debugging, i.e., the diagnoses, by checking consistency of derived or given predicates over a background theory. The background theory captures all knowledge about the domain of the variables, e.g., boolean algebra or arithmetic. In the following we assume that the required background theory is part of the program's model $\mathcal{D}$.

Definition 2 (Diagnosis). Given a program $\Pi$, the corresponding diagnosis problem $(\mathcal{D}, \mathcal{T})$, and a set of indices $\mathcal{I}$ where each $x \in \mathcal{I}$ corresponds to a certain statement of $\Pi$. A set $\Delta \subset \mathcal{I}$ is a diagnosis iff

$$
\mathcal{D} \cup \mathcal{T} \cup\{A b(x) \mid x \in \Delta\} \cup\{\neg A b(x) \mid x \in \mathcal{I} \backslash \Delta\}
$$

is consistent.

Since in most cases we are interested in diagnoses that are as small as necessary we introduce the concept of minimal diagnoses, i.e., diagnosis $\Delta$ is a minimal diagnosis if no proper subset $\Delta^{\prime} \subset \Delta$ is a diagnosis. As already mentioned by Reiter [9] there only exists a diagnosis if $\mathcal{D} \cup \mathcal{T}$ itself is consistent. Considering our model $\mathcal{D}$ from Figure 1 some other properties stated in [9] unfortunately do not hold. For example, it is not possible to remove $\{A b(x) \mid x \in \Delta\}$ and check consistency because $\mathcal{D}$ specifies the statement's behavior when assuming the statement to be faulty. Fortunately the following property still holds. 
Property 1. Every superset of a diagnosis is itself a diagnosis.

Assume a diagnosis $\Delta$ and a set $\Delta^{\prime} \supset \Delta$. For all elements in $\Delta^{\prime} \backslash \Delta$ suppose the assumption about their state changes from $\neg A b$ to $A b$. Because of the definition of rule (4) (Fig. 1) this change has an influence on the derived predicates. However, only less predicates may be derived. Therefore, it is still not possible to cause an inconsistency.

Because of Property 1 it is enough to compute all minimal diagnoses. However, computing all minimal diagnoses is still NP-complete because we have to check all subsets of $\mathcal{I}$. A better way of computing all minimal diagnosis up to a given size which works in practice is the following. Instead of computing diagnoses directly, we compute conflicts. These conflicts can be used directly to compute all diagnosis up to a given size.

Definition 3 (Conflict). Given a program $\Pi$, a corresponding diagnosis problem $(\mathcal{D}, \mathcal{T})$, and a set of indices $\mathcal{I}$ where each $x \in \mathcal{I}$ corresponds to a certain statement of $\Pi$. A set $\Xi \subset \mathcal{I}$ is a conflict iff

$$
\mathcal{D} \cup \mathcal{T} \cup\{\neg A b(x) \mid x \in \Xi\} \cup\{A b(x) \mid x \in \mathcal{I} \backslash \Xi\}
$$

is inconsistent.

The relationship between conflicts and diagnoses can be explained as follows. Assume we have two conflicts $\Xi_{1}=\{1,3\}$ and $\Xi_{2}=\{2,3\}$. This means that assuming statements 1,3 respectively 2,3 to be correct (and the others to be faulty) leads to an inconsistency. Formally, we can write $\ldots \cup\{\neg A b(1), \neg A b(3)\} \cup$ $\ldots \vdash \perp$ respectively $\ldots \cup\{\neg A b(2), \neg A b(3)\} \cup \ldots \vdash \perp$. In order to remove both conflicts we have to set the truth value of some $A b$ predicates to true which makes the literals $\neg A b$ to become false and the contradiction $\perp$ can no longer be derived. The algorithm for computing diagnosis from conflicts which is basically a hitting-set computation is described in [9, 4. This algorithm is also applicable in case of programs with multiple faults. Note that the computation of small conflicts can be improved by keeping track of the derivations. In most cases not all assumptions are involved in deriving an inconsistency.

\section{Model Checking and Counterexamples}

In order to compare our formal debugging model with an heuristic approach we briefly introduce the basic concepts of model checking and counterexamples [2]. The heuristic approach used for comparison stems from Groce and Visser [5].

Model checking of programs is based on a labeled transition system (LTS). An LTS is a 4-tuple $\left\langle S, S_{0}, A c t, T\right\rangle$, where $S$ is a finite non-empty set of states, $S_{0} \subset S$ is the set of initial states, Act is the set of actions, and $T \subset S \times A c t \times S$ is the transition relation. The program states in $S$ are composed of a control location $C$ and a data valuation $D$, and the partial functions $c: S \rightarrow C$ and $d: S \rightarrow D$ are defined. Furthermore the set of states $S$ contains a distinguished set of error states $\Pi=\left\{\pi_{1}, \ldots, \pi_{n}\right\}$ representing assertion violations, uncaught exceptions, etc. Hence, the set of states is written as $S=(C \times D) \cup \Pi$. The transition relations $\left(s, \alpha, s^{\prime}\right) \in T$ are written as $s \stackrel{\alpha}{\longrightarrow} s^{\prime}$. 
A finite transition sequence of length $k$ starting in state $s_{0} \in S_{0}$ is denoted as $t=s_{0} \stackrel{\alpha_{1}}{\longrightarrow} s_{1} \stackrel{\alpha_{2}}{\longrightarrow} \cdots \stackrel{\alpha_{k}}{\longrightarrow} s_{k}$, where $0<k<\infty$. A finite transition sequence $t=s_{0} \stackrel{\alpha_{1}}{\longrightarrow} s_{1} \stackrel{\alpha_{2}}{\longrightarrow} \cdots \stackrel{\alpha_{k}}{\longrightarrow} s_{k}$ is a prefix of the finite transition sequence $t^{\prime}=s_{0}^{\prime} \stackrel{\alpha_{1}^{\prime}}{\longrightarrow} s_{1}^{\prime} \stackrel{\alpha_{2}^{\prime}}{\longrightarrow} \cdots \stackrel{\alpha_{k^{\prime}}^{\prime}}{\longrightarrow} s_{k^{\prime}}^{\prime}$ if $0<k<k^{\prime}$ and $\forall i \leq k \cdot\left(i \geq 0 \Rightarrow s_{i}=s_{i}^{\prime}\right) \wedge(i>$ $0 \Rightarrow \alpha_{i}=\alpha_{i}^{\prime}$. Similarly a control suffix of transition sequence $t^{\prime}$ is a finite transition sequence $t=s_{0} \stackrel{\alpha_{1}}{\longrightarrow} s_{1} \stackrel{\alpha_{2}}{\longrightarrow} \cdots \stackrel{\alpha_{k}}{\longrightarrow} s_{k}$ if $0<k<k^{\prime}$ and $\forall i \leq k$. $(i \geq$ $\left.0 \Rightarrow c\left(s_{k-i}\right)=c\left(s_{k^{\prime}-i}^{\prime}\right)\right) \wedge\left(i>0 \Rightarrow \alpha_{k-i}=\alpha_{k-i}^{\prime}\right)$. Based on an LTS and finite transition sequences the term counterexample is defined formally:

Definition 4. A counterexample is a finite transition sequence $t$ in the LTS $\left\langle S, S_{0}\right.$, Act, $\left.T\right\rangle$, where $s_{0} \in S_{0}, s_{k} \in \Pi$, and $t=s_{0} \stackrel{\alpha_{1}}{\longrightarrow} s_{1} \stackrel{\alpha_{2}}{\longrightarrow} \cdots \stackrel{\alpha_{k}}{\longrightarrow} s_{k}$.

With respect to an initial counterexample $t$ a so-called set of negatives is defined. It contains all counterexamples for the given LTS that result in the same error state from the same control location and is denoted by neg(t) (or neg for short). The set is formally described by:

Definition 5. A negative (w.r.t. a particular counterexample $t$ ) is a finite transition sequence from $s_{0}^{\prime} \in S_{0}, t^{\prime}=s_{0}^{\prime} \stackrel{\alpha_{1}^{\prime}}{\longrightarrow} s_{1}^{\prime} \stackrel{\alpha_{2}^{\prime}}{\longrightarrow} \cdots \stackrel{\alpha_{k^{\prime}}^{\prime}}{\longrightarrow} s_{k^{\prime}}^{\prime}$, where $0<k^{\prime}<\infty$, such that (1) $c\left(s_{k-1}\right)=c\left(s_{k^{\prime}-1}^{\prime}\right) \wedge \alpha_{k}=\alpha_{k^{\prime}}^{\prime}$, and (2) $s_{k}=s_{k^{\prime}}^{\prime}$.

Similarly the set of positives is defined. It consists of executions that contain the statement immediately before the error but do not reach the error state and is denoted by $\operatorname{pos}(t)$ (or pos for short).

Definition 6. A positive (w.r.t. a particular counterexample $t$ ) is a finite transition sequence from $s_{0}^{\prime} \in S_{0}, t^{\prime}=s_{0}^{\prime} \stackrel{\alpha_{1}^{\prime}}{\longrightarrow} s_{1}^{\prime} \stackrel{\alpha_{2}^{\prime}}{\longrightarrow} \cdots \stackrel{\alpha_{k^{\prime}}^{\prime}}{\longrightarrow} s_{k^{\prime}}^{\prime}$, where $0<k<\infty$, such that (1) $c\left(s_{k-1}\right)=c\left(s_{k^{\prime}-1}^{\prime}\right) \wedge \alpha_{k}=\alpha_{k^{\prime}}^{\prime}$, (2) $s_{k^{\prime}}^{\prime} \notin \Pi$, and (3) $\forall t^{\prime \prime} \in \operatorname{neg}(t) \cdot t^{\prime}$ is not a prefix of $t^{\prime \prime}$.

In general the sets $n e g(t)$ and pos $(t)$ are potentially infinite. In order to keep the analysis tractable only subsets are generated. This requires that requirement 3 has to be relaxed: A positive must not be a prefix of any found negative. The algorithm for generating negatives and positives uses a model checker to explore backwards from a given counterexample. The search may be limited in case the state space is quite large or infinite. For a detailed definition and description of this algorithm refer to [5].

\section{Case Study}

The case study for the comparison of our formal approach and the heuristic approach is based on a small example program.

\subsection{Example Program}

The example program (Figure 2) used for a detailed explanation of the two approaches is an adaptation from Henzinger et al. [6]. A similar version of the 
$1 \quad$ public static int $\mathrm{LOCK}=0$;

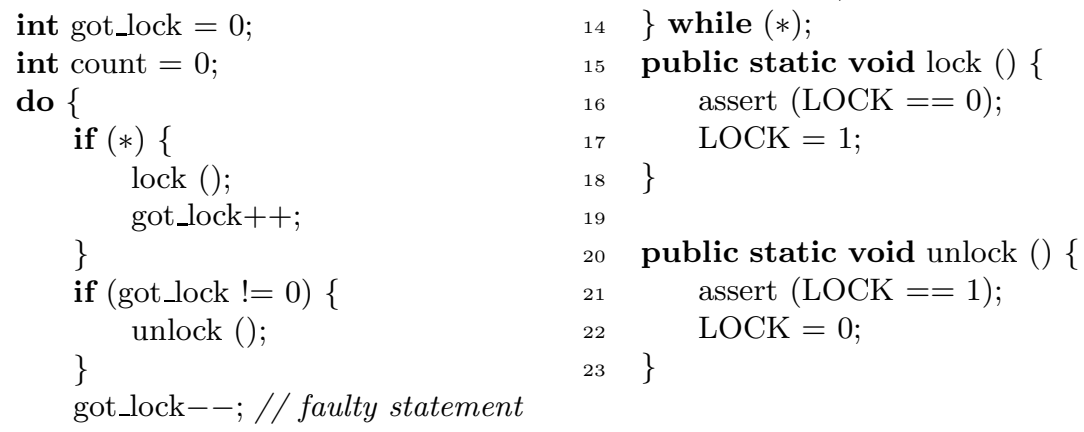

$14\}$ while $(*)$;

15 public static void lock () \{ assert $(\mathrm{LOCK}==0)$; $\mathrm{LOCK}=1$; \}

public static void unlock () \{ assert $(\mathrm{LOCK}==1)$; $\mathrm{LOCK}=0$;

13 count ++ ;

Fig. 2. Example program

program is also used by Groce and Visser [5]. The program uses methods lock() and unlock() in order to acquire and release a lock. The lock is represented by variable LOCK which is set to 0 or 1 according to the state of the lock. The two locking methods assert that the lock is not held already if it is going to be acquired and that it is held if it is going to be released. The program keeps track of the state of the lock with the additional variable got_lock. A $*$ in a condition of an if-statement or while-loop denotes a non-deterministic choice. A model checker that searches for violated assertions has to consider both possibilities.

The program in Figure 2 contains a fault with respect to its specification (i.e., the assertions). The decrement of variable got_lock in line 12 should be within the scope of the if-statement in line 9. This fault allows to acquire the lock twice and it is possible to release the lock although it is not held.

Compared to the version of the program used in [5] a third variable named count is added. It was introduced to force a larger state space that has to be explored by the model checker. The reason for this is that we want to evaluate the explaining counterexamples approach depending on the search depth. If variable count would be removed entirely from the program the state space explored by the model checker would be limited. Thus the number of positives and negatives would be limited as well.

\subsection{Explaining Counterexamples}

The work of Groce and Visser [5] is based on comparing negative and positive program traces. The analyses are intended to give an explanation on "what went wrong" in a given negative trace, i.e., a counterexample. Obviously this approach requires the existence of positive traces according to Definition 6. If no positives are found, the approach will not yield useful results.

The first analysis deals with sets of statements that occur in the negative and positive traces. Table 1 provides a definition of the sets that are used for the analysis. The interesting sets that are also given to the user are the only 
Table 1. Transition analysis set definitions

\begin{tabular}{|l|l|}
\hline Transition analysis set & Definitions \\
\hline \hline $\operatorname{trans}($ neg $)$ & $\langle c, \alpha\rangle \mid \exists t \in$ neg $\cdot t$ contains $\langle c, \alpha\rangle$ \\
$\operatorname{trans}($ pos $)$ & $\langle c, \alpha\rangle \mid \exists t \in$ pos $\cdot t$ contains $\langle c, \alpha\rangle$ \\
\hline all $($ neg $)$ & $\langle c, \alpha\rangle \mid \forall t \in$ neg $\cdot t$ contains $\langle c, \alpha\rangle$ \\
all $($ pos $)$ & $\langle c, \alpha\rangle \mid \forall t \in$ pos $\cdot t$ contains $\langle c, \alpha\rangle$ \\
\hline only $($ neg $)$ & trans $($ neg $) \backslash$ trans $($ pos $)$ \\
only $($ pos $)$ & trans $($ pos $) \backslash$ trans $($ neg $)$ \\
\hline cause $($ neg $)$ & all $($ neg $) \cap$ only $($ neg $)$ \\
cause $($ pos $)$ & all $($ pos $) \cap$ only $($ pos $)$ \\
\hline
\end{tabular}

and cause sets. For instance the set only $(n e g)$ denotes those statements that are unique to the negative traces, whereas the set cause (neg) contains those statements that are necessary for a trace to be negative. Note that the statements contained in any of the sets strongly depend on the negatives and positives found by the explanation algorithm. The size of the sets varies between almost all statements of the entire program and the empty set.

The second analysis deals with the transformation of a positive trace into a negative trace. Therefore this analysis focuses on the sequence of statements instead of sets of statements. A transformation from a positive trace $t$ into a negative trace $t^{\prime}$ is performed by replacing a block of statements occurring in $t$ with a block of statements occurring in $t^{\prime}$. Therefore it is necessary that traces $t$ and $t^{\prime}$ consist of the same prefix and the same control suffix. A transformation from $t=s_{0} \stackrel{\alpha_{1}}{\longrightarrow} s_{1} \stackrel{\alpha_{2}}{\longrightarrow} \cdots \stackrel{\alpha_{k}}{\longrightarrow} s_{k}$ into $t^{\prime}=s_{0}^{\prime} \stackrel{\alpha_{1}^{\prime}}{\longrightarrow} s_{1}^{\prime} \stackrel{\alpha_{2}^{\prime}}{\longrightarrow} \cdots \stackrel{\alpha_{k^{\prime}}^{\prime}}{\longrightarrow} s_{k^{\prime}}^{\prime}$ exists if

1. $\exists p \cdot p$ is a finite transition sequence which is a prefix of both $t$ and $t^{\prime}$, and

2. $\exists u \cdot u$ is a finite transition sequence which is a control suffix of both the largest prefix of $t$ and the largest prefix of $t^{\prime}$.

Note that in requirement 2 the largest prefix of transition sequences $t$ and $t^{\prime}$ are taken, since the two traces do not share the same final control location, that is the negative trace $t^{\prime}$ ends in an error state whereas the positive trace $t$ does not. The minimal transformation from $t$ to $t^{\prime}$ is defined as a 3 -tuple $\left\langle k_{t}, t_{p}, t_{n}\right\rangle$, where $k_{t}$ is the length of the prefix in $t\left(t^{\prime}\right)$, and $t_{p}\left(t_{n}\right)$ is the block of statements in $t$ $\left(t^{\prime}\right)$ that has to be replaced with $t_{n}\left(t_{p}\right)$ in order to get a negative (positive) trace. The statement blocks $t_{n}$ and $t_{p}$ are again evaluated with the transition analysis defined in Table 1 in order to extract causal information for the fault. Note that for the second analysis neg and pos in Table[1] are replaced with $t_{n}$ and $t_{p}$, respectively. Program traces are written as a sequence of statements, represented by their line number. Conditional statements containing a non-deterministic choice also contain the value that was chosen for the particular trace.

Our analysis is based on the counterexample with index 1 shown in Table 2 . The traces found by the model checker with a limited search depth of 17 transitions include a negative trace (1) which is the initial counterexample itself, a positive trace (2), a second negative (3), a trace that ends in an assertion but is 
Table 2. Traces found by model checker (search depth 17)

\begin{tabular}{|c|c|}
\hline Index & Trace \\
\hline 1 & 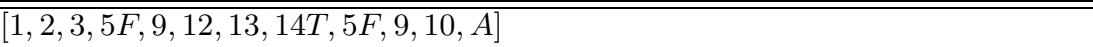 \\
\hline 2 & 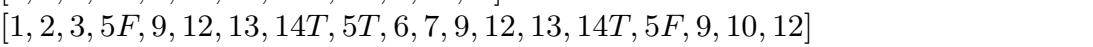 \\
\hline 3 & 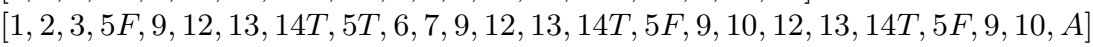 \\
\hline 4 & 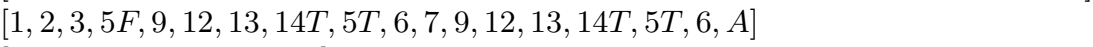 \\
\hline 5 & \begin{tabular}{|l|l|l|l|l|l|l|l|l|l|l|l|l|l|l|}
1 & 2 & 3 & 5 & 6 & 7 & 9 & 10 & 12 \\
\end{tabular} \\
\hline
\end{tabular}

not a valid negative (4), and a second positive trace (5). The first positive trace (2) has to be removed because it is a prefix of the second negative (3). Hence, the sets of negative and positive traces are $n e g=\{1,3\}$ and $p o s=\{5\}$.

Based on this information the transition analysis sets are computed. Table 3 depicts the resulting sets. These are the same results as described in 5] except that the increment of variable count occurs in some sets. This is not a problem since it is easily removed form the explanation by performing some dependency analysis such as slicing [10. Groce and Visser now conclude that the set cause =

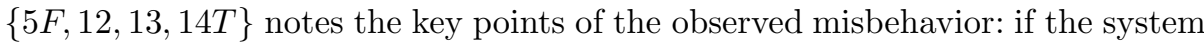
chooses not to lock $(5 F)$, the decrement of got_lock (12) together with the loop being reiterated (14T) leads to an erroneous state. Thus it is possible to try to unlock even though the lock has not been acquired.

This indeed is an interesting result as it clearly describes the cause of the observed misbehavior. Unfortunately this information depends on the positive and negative traces found by the model checker. If the search depth is increased or decreased, the results look quite different. For example a search depth of 18 causes the model checker to find an additional positive trace and the analysis sets change as can be seen in Table 4. The analysis now has identified the ifstatement in line 5 as the cause for the negatives. Unfortunately the real fault in the program is not present any more. The explanation that choosing false for the condition (i.e., not acquiring the lock) causes the error provides useful information to the user, but he still has to look at all statements that influence the locking/unlocking mechanism in order to locate the fault.

Even worse is the result of the analysis if the search depth is increased to 23. In this case the model checker identifies three negatives and four positives

Table 3. Transition analysis for search depth 17

\begin{tabular}{|c|c|}
\hline Analysis se & Elements \\
\hline trans (neg) & 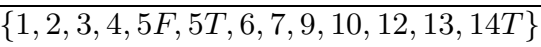 \\
\hline $\operatorname{trans}(p o s)$ & 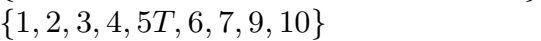 \\
\hline $\operatorname{all}(n e g)$ & $\left.\begin{array}{|l|l|l|l|l|l|l|l|l|l|l|}1 & 2 & 3 & 4 & 5 & F & 9 & 10 & 12 & 13 & 14\end{array}\right\}$ \\
\hline $\operatorname{all}(p o s)$ & 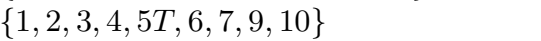 \\
\hline only $(n e g)$ & \begin{tabular}{|l|l|l|l|l|l|l|}
5 & 12 & 13 & 14 & $T$
\end{tabular} \\
\hline only $(p o s)$ & \{\} \\
\hline cause $($ neg $)$ & \begin{tabular}{|l|l|l|l|l|l|}
$5 F$ & 12 & 13 & 14 & $T$
\end{tabular} \\
\hline cause (pos) & \{\} \\
\hline
\end{tabular}


Table 4. Transition analysis for search depth 18

\begin{tabular}{|l|l|}
\hline Transition analysis set & Elements \\
\hline \hline $\begin{array}{l}\text { only }(\text { neg }) \\
\text { only }(\text { pos })\end{array}$ & $\{[5 F\}$ \\
\hline $\begin{array}{l}\text { cause }(\text { neg }) \\
\text { cause }(\text { pos })\end{array}$ & \{\} \\
\hline
\end{tabular}

Table 5. Transition analysis for search depth 6

\begin{tabular}{|c|c|}
\hline Transition & Elements \\
\hline only (neg) & \begin{tabular}{|l|l|l|l|l|l|}
5 & $F$ & 12 & 13 & 14 \\
\end{tabular} \\
\hline only $(p o s)$ & $\left.\begin{array}{|l|l|l|l|l|}\mid 5 & \Gamma & 6 & 7\end{array}\right\}$ \\
\hline $\begin{array}{l}\text { cause (neg) } \\
\text { cause (pos) }\end{array}$ & 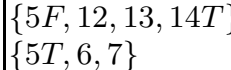 \\
\hline
\end{tabular}

Table 6. Transformation analysis results

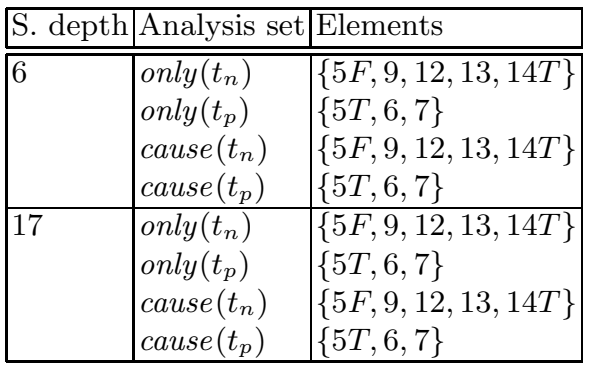

\begin{tabular}{|c|c|c|}
\hline \multicolumn{2}{|c|}{\begin{tabular}{l|l} 
S. depth & Analysis set
\end{tabular}} & Elements \\
\hline$\overline{18}$ & $\begin{array}{l}\operatorname{only}\left(t_{n}\right) \\
\operatorname{only}\left(t_{p}\right) \\
\operatorname{cause}\left(t_{n}\right) \\
\operatorname{cause}\left(t_{p}\right)\end{array}$ & 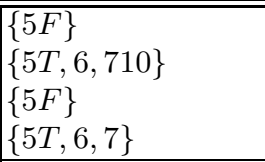 \\
\hline 23 & $\begin{array}{l}\operatorname{only}\left(t_{n}\right) \\
\text { only }\left(t_{p}\right) \\
\operatorname{cause}\left(t_{n}\right) \\
\operatorname{cause}\left(t_{p}\right)\end{array}$ & 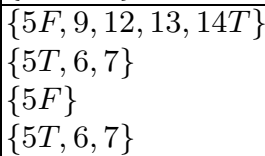 \\
\hline
\end{tabular}

whereof two are prefixes of some negative. The analysis reveals that it is not possible to identify any statement as a cause for the negatives or positives. Further increasing the search depth leads back to one of the above mentioned cases, that is no positives are found, the cause of negatives contains some statements, and the cause of negatives is empty. Furthermore if the search depth is less then 17 it is possible to identify a cause for positives that is given in Table 5. In this case the cause for positives is identified as taking the lock and incrementing variable got_lock. This set adds almost no new information since it only confirms that not taking the lock causes an error.

In addition to the transition analysis sets we also performed the transformation analysis. Table 6 collects the results of the analysis. The results are comparable to those of transition analysis. Table 6 also shows that even if transition analysis reports no causal sets it is still possible to get this information from transformation analysis (e.g., search depth 23). Although, the results are not improved by transformation analysis. The main proposition gathered from transition and transformation analysis is the fact that not taking the lock in line 5 causes an error.

\subsection{Applying Model-Based Debugging}

Computing possible fault locations for the given example program with MBD requires test cases. Contrary to model checking it is not possible to specify values non-deterministically, instead values have to be specified according to a specific program execution. For the given example program this means that 
Table 7. Diagnoses using different counterexamples

\section{(a) Counterexample 1}

\begin{tabular}{|l|}
\hline Diagnoses \\
\hline \hline$[$ LOCK $=0][1]$ \\
{$[$ got_lock $=0] 2$} \\
{$[$ if $(*)]$} \\
{$[$ if $($ got_lock ! $=0)]$} \\
$[$ unlock ()$] 10]$ \\
{$[$ got_lock --[]} \\
{$[$ while $(*)][1]$} \\
\hline
\end{tabular}

(b) Counterexamples 1 and 4

\begin{tabular}{|l|}
\hline Diagnoses \\
\hline \hline$[\text { if }(*)]_{5}$ \\
{$[$ if $($ got_lock ! $=0)$} \\
{$[$ got_lock --$]$} \\
{$[$ while $(*)][1]$} \\
\hline
\end{tabular}

(c) Counterexamples 1, 3, and 4

\begin{tabular}{|l|}
\hline Diagnoses \\
\hline \hline$[$ if $(*)]$ \\
{$[$ got_lock --$]$} \\
{$[$ while $(*)][14$} \\
\hline
\end{tabular}

the non-deterministic choices $(*)$ in lines [5] and 14 must be replaced with some expressions that enforce a predefined execution trace.

In order to compare the results of explaining counterexamples with the results of MBD the counterexamples found in Section 4.2 are translated into test cases. Since the example program uses neither input nor output variables, the test cases only include values for the conditions of the if-statement in line 5 and the while-statement in line 14. The assertion statements in lines 16 and 21 are also part of the model. Obviously, the resulting value of an assertion's expression is expected to be true. Therefore every test case will include an observation for assertions.

Table 7(a) depicts the set of diagnoses that is retrieved by applying MBD to the example program with the test case derived from counterexample 1 . Note that all diagnoses are single fault diagnoses. This means that every statement represented by a diagnosis has a direct influence on the observed misbehavior (i.e., the violated assertion in line 21). The set of diagnoses is rather big compared to the overall size of the example program. This particular result could also be reproduced using a dynamic slicing algorithm [10]. But we show that this result can be improved using multiple test cases.

The results of MBD can be improved by providing additional test cases because of their potential to introduce new conflicts which make diagnoses more concise. The model checker found two additional counterexamples, as is shown in Table 2. Trace 4 is most promising to improve the results since it is very different from the first one. Contrary to trace 1 it violates the assertion in method lock. Thus traces 1 and 4 are selected for MBD. Performing diagnosis with these two traces yields a smaller set of diagnoses (Table $7(\mathrm{~b})$. The resulting diagnoses now must be able to explain the fault in both traces. Thus, diagnoses that may only explain the fault in one of the two traces are removed. Note that Table $7(\mathrm{~b})$ only lists the single fault diagnoses, although there exist multiple fault diagnoses as well. But since multiple fault diagnoses are less likely to describe the real error they are omitted.

The set of diagnoses can be reduced slightly if the third counterexample, trace 3 , is also used for MBD. In this case the diagnosis containing the if-statement in line 9 is removed. Adding further counterexamples to the diagnostic process 
does not improve the results. The final set of diagnoses is shown in Table $7(\mathrm{c})$ This is the optimal result that we expect from MBD, since all three diagnoses that are left have a plausible explanation. It is possible to give a simple repair for each diagnosis that removes the fault with respect to the given specification. For example replacing the condition of the if-statement in line 5 with the constant true or the condition of the while-statement in line 14 with the constant false will prevent the violation of the assertions. Hence, it is not possible to get rid of these two diagnoses without any further specification. But most important the real fault (i.e., line 12) is among the diagnoses as well.

Considering the formal debugging framework based on resolution calculus the optimal result in terms of a minimal set of program statements has been found. It is not possible to improve the above result in terms of improving the diagnostic process or the used model. But still the result can be enhanced before it is presented to the user. For example it is very likely that conditional statements are part of the set of diagnoses. This stems from the way they are modeled for diagnosis. A conditional statement is responsible for selecting one of the possible branch values for each variable that is part of the conditional statement block. Therefore a conditional statement can rarely be removed from the diagnoses. Hence, a way to further improve MBD results is the application of some rating mechanism. Every type of diagnosis is assigned some probability value. The user would then be able to check the most likely diagnoses first. Obviously this is a heuristic approach that requires a well-founded analysis on the likelihood of faults in software systems.

The time consumed by the two approaches is quite similar, if we account searching for the positive and negative traces to the fault explanation approach as well. We conducted our evaluation on a Pentium 4 with $2.66 \mathrm{GHz}$ using Java PathFinder (JPF) and a prototypical implementation of the value-based diagnosis model. Computing diagnoses for the example program in Figure2 takes about 0.24 seconds on average and 2.4 seconds with overhead for parsing and model generation. The time for computing the initial counterexample with JPF takes 1.6 seconds on average. The time to search for additional negative and positive traces and computing the analysis sets is about 3.0 seconds. For larger programs, for instance a search tree program with approx. 150 lines of code using several insertion, deletion and search operations, diagnosis time ranges from 1 up to 10 seconds. The running time depends on the type and the location of a fault. With JPF the first counterexample was detected after 2.5 seconds on average. The analysis then lasted 3.5 seconds on average. Note that for the search tree example we provided a set of test cases for the model checker. Thus it was not necessary to search in a large state space to locate a counterexample.

\section{Related Research and Conclusion}

Software debugging and the application of automated tools to this task is an active research area. Detecting bugs using model checking [2] is becoming an accepted technique, providing witnesses of bugs called counterexamples and cor- 
responding test cases. Several solutions to the challenge of localizing bugs based on these witnesses were proposed. Especially the model checking community applies heuristic approaches for localization. Common to all these approaches is their lack of a clear semantics for the reported fault locations.

In addition to the explanation approach discussed in this paper, Ball et al. [1] for instance use a model checker to identify the cause of an error. Their approach searches for counterexamples and compares them to a correct trace of the program. The main idea is that the differences between correct traces and erroneous traces identify the root cause of a fault. Those parts of the counterexample that are not part of the correct trace are replaced with halt-instructions. This prevents the model checker in subsequent runs to find the same counterexamples again. The additional counterexamples are used to refine the fault locations.

A similar work to fault explanation is the work of Zeller et al. [7, 12, Delta debugging. The motivation of their work is to answer the question why a program does not work anymore after changing it. The aim of delta debugging is to isolate a cause-effect chain that represents the variables and their values that caused the failure. Based on a failing and a correct program execution, delta debugging minimizes the difference between the two runs in terms of isolating the relevant input and states. Analogous to explaining counterexamples this approach relies on the existence of successful program runs that are similar to the failing ones.

Model-based diagnosis is related to program-slicing [10] as is stated by Wotawa [1]. In case of a dependency model for diagnosis it is shown that slices are equivalent to conflict sets. In our debugging calculus the relation to slicing appears in terms of Hoare's rules, since a predicate is only transformed by a statement if it has an effect on the predicate. This is similar to the slicing approach using weakest preconditions proposed by Comuzzi and Hart [3] .

We presented a formalization of model-based debugging based on a resolution calculus. Contrary to other debugging approaches we provide a formal model for describing program behavior and do not rely on any heuristics. The formality of the model and the underlying diagnosis theory allows to assign a precise semantics to the diagnoses (i.e., fault locations) reported by the approach. This is an essential difference to heuristic approaches where no clear semantics for a reported cause is given. The quality of heuristic approaches diverges depending on many parameters. The same fault produces different causes depending on the position of the fault within the program, the program traces used for explanation and the time spent for explanation (i.e., the search depth). Contrary, our approach does not make any structural assumptions except the implicit fault model. Adding more information (e.g., more counterexamples) leads to more precise diagnoses, whereas the results of heuristic approaches may become worse.

The presented approach contains some limitations. The debugging calculus used to model program behavior induces a specific fault model. That is, statements that are assumed to be faulty do not affect predicates that do not contain any target variable of that statement. This fault model restricts the set of faults that are precisely localizable with our approach. In case of a faulty assignment statement with the wrong target variable our approach derives wrong predi- 
cates. Nevertheless, debugging experiments based on this calculus have shown that such structural faults are localized by our approach, but only in diagnoses with higher cardinality. This stems from the fact that a wrong target variable in general provokes a wrong value in two variables, the actual target variable and the intended target variable.

Faults that result in non-terminating programs can not be handled at all. This mainly stems from modeling of loop statements by unrolling them into nested conditionals. The number of loop iterations must be known to generate the behavioral model of a program.

\section{References}

1. T. Ball, M. Naik, and S. K. Rajamani. From Symptom to Cause: Localizing Errors in Counterexample Traces. In Proceedings of the $30^{\text {th }}$ ACM SIGPLAN-SIGACT Symposium on Principles of Programming Languages (POPL'03), volume 38, 1 of ACM SIGPLAN Notices, pages 97-105, Jan. 2003.

2. E. M. Clarke, O. Grumberg, and D. A. Peled. Model Checking. The MIT Press, Cambridge, Massachusetts, 1999.

3. J. J. Comuzzi and J. M. Hart. Program Slicing using Weakest Preconditions. Lecture Notes in Computer Science, 1051:557-575, 1996.

4. R. Greiner, B. A. Smith, and R. W. Wilkerson. A Correction to the Algorithm in Reiter's Theory of Diagnosis. Artificial Intelligence, 41(1):79-88, 1989.

5. A. Groce and W. Visser. What Went Wrong: Explaining Counterexamples. In SPIN Workshop on Model Checking of Software, pages 121-135, May 2003.

6. T. A. Henzinger, R. Jhala, R. Majumdar, and G. Sutre. Lazy Abstraction. In Proceedings of the 29 $9^{\text {th }}$ ACM SIGPLAN-SIGACT Symposium on Principles of Programming Languages (POPL'02), volume 37, pages 58-70, Jan. 2002.

7. R. Hildebrandt and A. Zeller. Simplifying Failure-Inducing Input. SIGSOFT Software Engineering Notes, 25(5):135-145, 2000.

8. C. A. R. Hoare. An Axiomatic Basis for Computer Programming. Communications of the ACM, 12(10):576-583, 1969.

9. R. Reiter. A Theory of Diagnosis from First Principles. Artificial Intelligence, 32(1):57-95, 1987.

10. M. Weiser. Program slicing. IEEE Transactions on Software Engineering, 10(4):352-357, July 1984.

11. F. Wotawa. On the Relationship between Model-Based Debugging and Program Slicing. Artificial Intelligence, 135(1-2):124-143, 2002.

12. A. Zeller. Isolating Cause-Effect Chains from Computer Programs. In ACM SIGSOFT 10th International Symposium on the Foundations of Software Engineering (FSE-10), pages 1-10, Charleston, South Carolina, Nov. 2002. 